\title{
Densidad seca al aire y características cuantitativas de elementos xilemáticos de conducción en 10 especies de Malvaceae en Venezuela
}

\section{Air dry density and quantitative features of conduction xylem elements in 10 species of Malvaceae in Venezuela}

\author{
Mariangel Uzcátegui-Rojas ${ }^{1}$, Styles W. Valero², \\ Williams J. León-Hernández ${ }^{3}$
}

Fecha de recepción: 28 de octubre de 2020

Fecha de aprobación: 10 de febrero de 2021

Uzcátegui-Rojas, M; Valero, S.W; León-Hernández, W.J.

Densidad seca al aire y características cuantitativas de elementos xilemáticos de conducción en 10 especies de Malvaceae en Venezuela. Tecnología en Marcha. Vol. 35-1. EneroMarzo 2022. Pág 14-27.

doi) https://doi.org/10.18845/tm.v35i1.5436

1 Universidad de Los Andes. Facultad de Ciencias Forestales y Ambientales. Laboratorio de Anatomía de Maderas.

Correo electrónico: mjuruzcateguir@gmail.com

(iD https://orcid.org/0000-0002-7560-8235

2 Universidad de Los Andes. Facultad de Ciencias Forestales y Ambientales. Laboratorio Nacional de Productos Forestales. Sección de Ensayos. Correo electrónico: styles@ula.ve 


\title{
Palabras clave
}

Anatomía madera; densidad madera; relación; propiedades físicas; vasos.

\section{Resumen}

Se presenta el estudio de la relación entre la densidad seca al aire y características cuantitativas de los vasos (frecuencia y diámetro de vasos, área de lumen) para diez especies de Malvaceae que crecen en Venezuela: Catostemma commune, Eryotheca globosa, Ochroma pyramidale, Pachira quinata, Guazuma ulmifolia, Apeiba tibourbou, Heliocarpus americanus, Uladendron codesurii, Sterculia apetala y Sterculia pruriens. La densidad se determinó en muestras acondicionadas al $12 \%$ de humedad y para la medición de las características de los vasos se prepararon láminas de secciones transversales utilizando los procedimientos convencionales de microtecnia xilemática. Las mayores frecuencias y menores diámetros de vasos se presentaron en Guazuma ulmifolia y Uladendron codesurii; mientras que las menores frecuencias y mayores diámetros de vasos los presentaron especies de las subfamilias Bombacoideae y Sterculioideae. La densidad seca al aire mostró un alto grado de variación, encontrándose especies que van desde la categoría de muy baja densidad (Ochroma pyramidale, Pachira quinata, Apeiba tibourbou, Heliocarpus americanus, Sterculia apetala) hasta muy alta densidad (Uladendron codesurii). La relación entre la densidad y las características de los vasos presentó una baja correlación lo que indica la posible existencia de otras características anatómicas que ejercen mayor influencia sobre esta propiedad física.

\section{Keywords}

Wood anatomy; wood density; relationship; physical properties; vessels.

\begin{abstract}
This research is about the relation between air dry density and quantitative features of vessels (frequency and diameter, lumen area) for ten species of Malvaceae from Venezuela: Catostemma commune, Eryotheca globosa, Ochroma pyramidale, Pachira quinata, Guazuma ulmifolia, Apeiba tibourbou, Heliocarpus americanus, Uladendron codesurii, Sterculia apetala and Sterculia pruriens. Density was determined in samples with moisture content equal to $12 \%$ and measures of vessels features were realized in slides samples of cross sections using standards procedures in xylem microtechnique. Higher vessels frequencies and lower diameters were observed in Guazuma ulmifolia and Uladendron codesurii and lower frequencies and higher diameters were observed in species from Bombacoideae and Sterculioideae subfamilies. Air dry density shows a high variation, ranging from very low density (Ochroma pyramidale, Pachira quinata, Apeiba tibourbou, Heliocarpus americanus, Sterculia apetala) to very high density (Uladendron codesurii). A low correlation between density and vessels features was found and its possible that others wood anatomy features have a higher effect over wood density for Malvaceae.
\end{abstract}

\section{Introducción}

La densidad de la madera es una de sus propiedades físicas más importantes ya que es indicativo de su comportamiento en otras propiedades físico-mecánicas. Según [1] la densidad es la expresión física de algunas características anatómicas. Tomando en consideración que la densidad o peso específico representan una medida de la cantidad total de material de la pared celular disponible por unidad de volumen, esto indica que está relacionada con las 
proporciones relativas de los diferentes tipos celulares así como también con las dimensiones de esas células y el grosor de sus paredes [2]. En el caso de las angiospermas, la alta variación en cuanto a tipos de células y proporción que ocupan dentro del tejido leñoso hace necesario determinar la posible influencia de los tipos celulares sobre la densidad o peso específico de la madera. Según [2], generalmente, la mayor cantidad de espacios vacíos en la madera de angiospermas está representada por los vasos y características tales como diámetro, frecuencia, porosidad y proporción de vasos pueden jugar un papel importante sobre la densidad o peso específico. En años recientes, los cambios taxonómicos que se han producido a nivel de la familia Malvaceae a través del sistema [3], [4], [5] han implicado una reorientación de diversas investigaciones para buscar conocer el efecto de esta nueva agrupación en aspectos diferentes al taxonómico. Algunas especies de Malvaceae en Venezuela son de importancia en el sector forestal y, durante algún tiempo, fueron la principal fuente de materia prima para la industria del procesamiento mecánico de la madera tal como es el caso de Pachira quinata, especie que hoy se encuentra bajo medidas de protección para garantizar su permanencia en el bosque. Otras especies de importancia forestal dentro de esta familia son Ceiba pentandra, Ochroma pyramidale, Sterculia pruriens. En la actualidad, aún cuando el mayor porcentaje de la madera que llega al mercado maderero nacional corresponde a Pinus caribaea var. hondurensis; algunas Malvaceae forman parte del grupo de especies que son aprovechadas por la Empresa Nacional Forestal (ENAFOR): Catostemma commune, Ceiba pentandra, Eriotheca globosa y Sterculia pruriens. Hasta hace poco, las especies mencionadas eran incluidas dentro de otras familias botánicas y esta nueva agrupación hace necesario realizar estudios que ayuden a tener una visión tecnológica de lo que corresponde a Malvaceae según el sistema [3], [4], [5]. Esta visión debe tener como punto de partida el estudio de una de las propiedades físicas más importantes: la densidad, propiedad que además de ser un índice con valor predictivo sobre otras propiedades de la madera, se utiliza como criterio para su clasificación en grupos de maderas blandas, moderadamente duras y duras. En Venezuela, en relación a la madera, el primer paso investigativo para el nuevo concepto de Malvaceae es el realizado por [6] con el estudio de la estructura anatómica en 25 especies de la familia en cuestión. Con respecto a estudios de tecnología de la madera y, específicamente, en cuanto a propiedades físicas, no se tiene ninguna información para Malvaceae sensu stricto en el país. Existen algunos datos para especies que formaban parte de las familias Bombacaceae, Sterculiaceae y Tiliaceae; pero con esta nueva agrupación no se conoce el rango de amplitud de los valores de propiedades como la densidad dentro de la Malvaceae.

En relación a propiedades de la madera, para la familia Malvaceae en Venezuela se encuentran diferentes investigaciones como las realizadas para Catostemma commune [7], Ochroma pyramidale [8], Ceiba pentandra [8], Pachira quinata [9], [8] y Eriotheca globosa [10]. Con respecto a la anatomía de maderas de Malvaceae, la única investigación bajo el nuevo concepto de esta familia es la presentada por [6]. En Venezuela se han realizado investigaciones de anatomía xilemática para lo que en otros sistemas de clasificación eran Bombacaceae [11] y Tiliaceae [12]. Con respecto a la Malvaceae sensu stricto, el único reporte es la descripción de Uladendron codesurii [13]. En algunas investigaciones donde se presentan descripciones de especies pertenecientes a diferentes grupos taxonómicos se incluyen algunas de lo que eran las familias Bombacaceae, Sterculiaceae y Tiliaceae [14], [15], [16], [17], [18] y [19]. En el establecimiento de relación entre anatomía y propiedades de la madera, se tienen investigaciones para algunas angiospermas [20], [21], [22], [23], [24], [25], [26], [27], [28], [29], [30], [31] y [32]. En Venezuela se pueden mencionar las realizadas por [33], [34], [35] y [2]. Dentro de este gran cúmulo de información, no hay reportes con relación a la anatomía y propiedades de la madera para Malvaceae. 
Tomando en consideración la importancia de la densidad como propiedad de la madera y la necesidad de conocer su variación dentro de los diferentes grupos taxonómicos, se plantea iniciar las investigaciones en Malvaceae con diez especies que incluyan antiguos representantes de Bombacaceae, Sterculiaceae, Tiliaceae y Malvaceae sensu stricto. Adicionalmente, se busca establecer la relación de los valores de densidad con los valores de las características dimensionales de los elementos xilemáticos de conducción.

\section{Materiales y métodos}

El material de estudio corresponde a muestras de madera de diez especies (cuadro 1) de la familia Malvaceae, agrupadas por subfamilias, colectadas en diferentes regiones geográficas de Venezuela y que pertenecen a la colección permanente de la xiloteca MERw del Laboratorio de Anatomía de Maderas de la Facultad de Ciencias Forestales y Ambientales de la Universidad de Los Andes, Mérida, Venezuela.

El número de individuos por especie osciló entre dos y cinco dependiendo de la cantidad de material disponible en la xiloteca antes mencionada. De cada muestra se extrajeron secciones para la realización del estudio de las características cuantitativas de los vasos (frecuencia, diámetro) y la densidad de la madera.

La determinación de la densidad se realizó en la Sección de Ensayos del Laboratorio Nacional de Productos Forestales, adscrito a la Facultad de Ciencias Forestales y Ambientales de la Universidad de Los Andes y al Ministerio del Poder Popular para el Ecosocialismo. De cada individuo se extrajeron diez probetas de, aproximadamente, $2 \mathrm{~cm} \times 2 \mathrm{~cm} \times 0,5 \mathrm{~cm}$ y se llevaron al cuarto de acondicionamiento (humedad de $65 \pm 2 \%$; temperatura de $20 \pm 1{ }^{\circ} \mathrm{C}$ ) para su respectiva aclimatación hasta alcanzar el contenido de humedad de equilibrio $(\mathrm{CH}=12 \%)$. Para la determinación de la densidad seca al aire se siguió el procedimiento indicado por la Norma DIN 52182 [36]. Se determinó el peso y el volumen, por el método de desplazamiento de agua, de la muestra en una balanza digital marca AND EK - 1200A con precisión de 0,1 g. La densidad seca al aire se calculó utilizando la ecuación 1.

$\rho=\frac{\text { Peso de la madera a un } C \cdot H \cdot(12 \%)}{\text { Volumen de la madera al mismo } C \cdot H \cdot(12 \%)}\left(\mathrm{kg} / \mathrm{m}^{3}\right)$

El valor promedio obtenido de la densidad seca al aire se utilizó para la posterior clasificación de las especies estudiadas de acuerdo a los criterios establecidos por [37] (cuadro 2). 
Cuadro 1. Especies estudiadas de Malvaceae, muestra de xiloteca y herbario.

\begin{tabular}{|c|c|c|}
\hline Especie & Muestra xiloteca & $\mathrm{N}^{\circ}$ muestra de herbario y/o colector \\
\hline \multicolumn{3}{|c|}{ Subfamilia Bombacoideae } \\
\hline \multirow[t]{4}{*}{ Catostemma commune Sandwith. } & $\mathrm{X} 1818$ & 3841 Breteler \\
\hline & X5594 & V1201 Bo Hernández y León H. \\
\hline & X5595 & V1202Bo Hernández y León H. \\
\hline & X5596 & V1203Bo Hernández y León H. \\
\hline \multirow[t]{4}{*}{ Eryotheca globosa Schott \& Endl. } & X3575 & 75 Conejos \\
\hline & X7079 & * \\
\hline & X7084 & * \\
\hline & X7085 & * \\
\hline \multirow[t]{4}{*}{ Ochroma pyramidale (Cav. ex Lam.) Urb. } & $\mathrm{x} 29$ & 15082 Little \\
\hline & X3704 & P. E. 70 \\
\hline & X6696 & 54549 J. Parra \\
\hline & X6701 & $54548 \mathrm{~J}$. Parra \\
\hline \multirow[t]{4}{*}{ Pachira quinata (Jacq.) W. S. Alverson } & $\mathrm{X} 1177$ & 361 Ruíz Terán \\
\hline & X4518 & 2 H. Gutiérrez \\
\hline & X4520 & 65 H. Gutiérrez \\
\hline & $\times 4521$ & 74 H. Gutiérrez \\
\hline \multicolumn{3}{|c|}{ Subfamilia Byttneroideae } \\
\hline \multirow[t]{5}{*}{ Guazuma ulmifolia Lam. } & X4997 & 103 L. Valverde \\
\hline & X6283 & 53167 L. Gámez \\
\hline & X6284 & 53168 L. Gámez \\
\hline & X6285 & 53169 L. Gámez \\
\hline & X6286 & 53170 L. Gámez \\
\hline \multicolumn{3}{|c|}{ Subfamilia Grewioideae } \\
\hline \multirow{4}{*}{ Apeiba tiborbou Aubl. } & X5569 & 1294 L. Valverde \\
\hline & X5570 & 1295 L. Valverde \\
\hline & X5571 & 1296 L. Valverde \\
\hline & X5572 & 1300 L. Valverde \\
\hline \multirow[t]{4}{*}{ Heliocarpus americanus L. } & X2640 & 235 Bautista \\
\hline & X6109 & 52985 L. Rondón \\
\hline & X5580 & 1307 L. Valverde \\
\hline & X5581 & 1308 L. Valverde \\
\hline \multicolumn{3}{|c|}{ Subfamilia Malvoideae } \\
\hline \multirow[t]{2}{*}{ Uladendron codesurii Marcano-Berti. } & X3634 & 2565 Marcano Berti \\
\hline & X3739 & 11629 L. Williams \\
\hline \multirow{5}{*}{ Sterculia apetala (Jacq.) H. Karst. } & Subfamilia Sterculio & \\
\hline & $x 5649$ & V09 01 Ba Hernández y León H. \\
\hline & X5650 & V09 02 Ba Hernández y León H. \\
\hline & X5651 & V09 03 Ba Hernández y León H. \\
\hline & $\times 5652$ & V09 04 Ba Hernández y León H. \\
\hline \multirow[t]{4}{*}{ Sterculia pruriens (Aubl.) K. Schum. } & X2573 & 165 Marcano Berti \\
\hline & X5247 & 91-981 Marcano Berti \\
\hline & X7081 & * \\
\hline & X7083 & * \\
\hline
\end{tabular}


Cuadro 2. Categorías de densidad seca al aire [37] Frecuencia y diámetro de poros [38].

\begin{tabular}{|c|c|c|c|}
\hline Categoría & Densidad $\left(\mathrm{kg} / \mathrm{m}^{3}\right)$ & Poros $/ \mathrm{mm}^{2}$ & Diámetro de poros $(\mu \mathrm{m})$ \\
\hline I & Muy Baja: $<500$ & $<5$ & $<50$ \\
\hline II & Baja: $500-660$ & $5-20$ & $50-100$ \\
\hline III & Media: $661-849$ & $20-40$ & $100-200$ \\
\hline IV & Alta: $850-1000$ & $40-100$ & $>200$ \\
\hline V & Muy alta: $>1000$ & $>100$ & -- \\
\hline
\end{tabular}

Fuente: Adaptación de [37] y [38].

La determinación de las características cuantitativas de los elementos xilemáticos de conducción se realizó en el Laboratorio de Anatomía de Maderas de la Facultad de Ciencias Forestales y Ambientales de la Universidad de Los Andes (Mérida, Venezuela). Cada muestra se sometió a un proceso de ablandamiento en agua hirviendo (10 min - 60 horas dependiendo de la dureza de la madera), se obtuvieron secciones transversales de 25 - 30 $\mu$ m de espesor mediante la realización de cortes con un micrótomo de deslizamiento; a cada sección se le aplicó un proceso de tinción con safranina (2 - 4 h), deshidratación en baños sucesivos de alcohol (50 \%, $10 \mathrm{~min} ; 70$ \%, $10 \mathrm{~min} ; 75$ \%,10 min; $95 \%$, 1 h) y el respectivo aclaramiento con una mezcla alcohol-xilol (1:1; $30 \mathrm{~min})$ y xilol $(1-2 \mathrm{~h})$ para luego hacer el montaje con resina sintética y el correspondiente rotulado.

Las mediciones se realizaron siguiendo los criterios establecidos por [38] en cuanto a procedimiento y número de mediciones, haciendo uso de un microscopio óptico marca Leica, modelo Galen III. Tanto para la frecuencia como el diámetro de poros, las especies se ubicaron en las categorías indicadas por [38] de acuerdo a los valores promedio observados en cada muestra (cuadro 2).

Con los valores de frecuencia y diámetro de vasos se calculó la fracción del lumen de los vasos de acuerdo a la fórmula 2 por [39]:

$\mathrm{Flv}=F p x D p\left(\mu \mathrm{m} \times \mathrm{mm}^{2}\right)$

Donde:

$F / V=$ fracción de lumen de vasos; $F p=$ frecuencia de poros $\left(\right.$ poros $\left./ \mathrm{mm}^{2}\right)$;

$D p=$ diámetro promedio de poros $(\mu m)$.

Tanto para la densidad como para las características de los vasos, se determinó la estadística descriptiva (mínimo, promedio, máximo, desviación estándar, coeficiente de variación) y se realizó un análisis de varianza y la respectiva prueba de medias (Tukey, a =0,01) para determinar si existen diferencias estadísticamente significativas entre las especies estudiadas. Para la relación anatomía y densidad se aplicó una correlación de Pearson. Dichos análisis estadísticos se llevaron a cabo mediante el software SPSS 22.0 Statistics.

\section{Resultados}

A continuación se presenta el análisis comparativo entre las especies de cada subfamilia (cuadro 3). 
Cuadro 3. Frecuencia de poros, diámetro de poros y densidad seca al aire (promedio, desviación, coeficiente de variación, número de mediciones) para especies de la familia Malvaceae agrupadas y comparadas dentro de cada subfamilia.

\begin{tabular}{|c|c|c|c|}
\hline Especie & Poros $/ \mathrm{mm}^{2}$ & Diámetro de poros $(\mu \mathrm{m})$ & Densidad seca al aire $\left(\mathrm{kg} / \mathrm{m}^{3}\right)$ \\
\hline \multicolumn{4}{|c|}{ Subfamilia Bombacoideae } \\
\hline $\begin{array}{l}\text { Catostemma } \\
\text { commune }\end{array}$ & $\begin{array}{c}4,85^{b} \pm 1,32 \\
C V=27,22 \% ; n=20\end{array}$ & $\begin{array}{c}227,22^{b} \pm 38,23 \\
C V=16,82 \% ; n=100\end{array}$ & $\begin{array}{c}682^{d} \pm 90 \\
C V=13,2 \% ; n=40\end{array}$ \\
\hline Eriotheca globosa & $\begin{array}{c}3,42^{a, b} \pm 1,79 \\
C V=52,34 \% ; n=20\end{array}$ & $\begin{array}{c}229,4^{b} \pm 49,82 \\
C V=21,72 \% ; n=100\end{array}$ & $\begin{array}{c}607^{\circ} \pm 60 \\
C V=9,88 \% ; n=40\end{array}$ \\
\hline $\begin{array}{l}\text { Ochroma } \\
\text { pyramidale }\end{array}$ & $\begin{array}{c}3,04^{\mathrm{a}} \pm 1,7 \\
\mathrm{CV}=55,92 \% ; n=20\end{array}$ & $\begin{array}{c}193,55^{\mathrm{a}} \pm 29,97 \\
C V=15,48 \% ; n=100\end{array}$ & $\begin{array}{c}185^{a} \pm 34 \\
C V=18,38 \% ; n=36\end{array}$ \\
\hline Pachira quinata & $\begin{array}{c}3,13^{a} \pm 1,41 \\
C V=45,05 \% ; n=20\end{array}$ & $\begin{array}{c}286,45^{\circ} \pm 62,18 \\
C V=21,71 \% ; n=100\end{array}$ & $\begin{array}{c}436^{b} \pm 94 \\
C V=21,56 \% ; n=40\end{array}$ \\
\hline \multicolumn{4}{|c|}{ Subfamilia Byttneroideae } \\
\hline Guazuma ulmifolia & $\begin{array}{c}18,52 \pm 11,07 \\
C V=59,78 \% ; n=25\end{array}$ & $\begin{array}{c}146,68 \pm 24,67 \\
C V=16,82 ; n=125\end{array}$ & $\begin{array}{c}612 \pm 61 \\
C V=9,92 \% ; n=50\end{array}$ \\
\hline \multicolumn{4}{|c|}{ Subfamilia Grewioideae } \\
\hline Apeiba tibourbou & $\begin{array}{c}4,56^{b} \pm 1,43 \\
C V=31,36 \% ; n=20\end{array}$ & $\begin{array}{c}170,85^{b} \pm 28,81 \\
C V=16,86 \% ; n=100\end{array}$ & $\begin{array}{c}400^{\mathrm{b}} \pm 50 \\
\mathrm{CV}=12,5 \% ; n=20\end{array}$ \\
\hline $\begin{array}{l}\text { Heliocarpus } \\
\text { americanus }\end{array}$ & $\begin{array}{c}9,31^{\mathrm{a}} \pm 6,85 \\
C V=73,58 \% ; n=20\end{array}$ & $\begin{array}{c}158,92^{a} \pm 29,98 \\
C V=18,86 \% ; n=100\end{array}$ & $\begin{array}{c}230^{\mathrm{a}} \pm 43 \\
C V=18,69 \% ; n=20\end{array}$ \\
\hline \multicolumn{4}{|c|}{ Subfamilia Malvoideae } \\
\hline $\begin{array}{l}\text { Uladendron } \\
\text { codesurii }\end{array}$ & $\begin{array}{c}8,96 \pm 2,76 \\
C V=30,82 \% ; n=20\end{array}$ & $\begin{array}{c}129,45 \pm 23,27 \\
C V=17,97 \% ; n=100\end{array}$ & $\begin{array}{c}1037 \pm 100 \\
C V=9,62 \% ; n=11\end{array}$ \\
\hline \multicolumn{4}{|c|}{ Subfamilia Sterculioideae } \\
\hline Sterculia apetala & $\begin{array}{c}4,04^{a} \pm 1,72 \\
C V=42,57 \% ; n=20\end{array}$ & $\begin{array}{c}240,25^{a} \pm 29,85 \\
C V=12,42 \% ; n=100\end{array}$ & $\begin{aligned} 457^{a} \pm 41 \\
C V=8,97 \% ; n=40\end{aligned}$ \\
\hline Sterculia pruriens & $\begin{aligned} & 4,37^{a} \pm 2,32 \\
C V= & 53,09 \% ; n=20\end{aligned}$ & $\begin{array}{c}238,9^{a} \pm 45,76 \\
C V=19,15 \% ; n=100\end{array}$ & $\begin{aligned} & 597^{\mathrm{b}} \pm 52 \\
C V= & 8,71 \% ; n=40\end{aligned}$ \\
\hline
\end{tabular}

NOTA: Letras diferentes indican grupos con diferencias estadísticas significativas (Tukey, $a=0,01$ ).

A nivel de la subfamilia Bombacoideae se observa poca diferencia entre las especies en lo que se refiere a frecuencia de poros (cuadro 3), los promedios oscilan entre 3,04 poros $/ \mathrm{mm}^{2}$ (Ochroma pyramidale) y 4,85 poros $/ \mathrm{mm}^{2}$ (Catostemma commune) y las cuatro especies se ubican en la categoría I de [38]. Con respecto al diámetro de los poros (cuadro 3), el menor promedio correspondió a Ochroma pyramidale y el mayor a Pachira quinata; tres especies se ubicaron en la categoría IV y una en la categoría III. Las mayores similitudes en cuanto a frecuencia y diámetro de poros se observaron en Catostemma commune y Eriotheca globosa. Los valores de densidad mostraron una alta variación: desde $185 \mathrm{~kg} / \mathrm{m}^{3}$ (Ochroma pyramidale) hasta $682 \mathrm{~kg} / \mathrm{m}^{3}$ (Catostemma commune). De acuerdo a las categorías establecidas por [37], dos especies se ubican en la categoría de muy baja densidad (Ochroma pyramidale, Pachira quinata), una en la de densidad baja (Eriotheca globosa) y una en la de densidad media (Catostemma commune); de acuerdo al análisis estadístico (Tukey, $a=0,01$ ) las cuatro especies se ubican en cuatro 
grupos diferentes, incluyendo las dos especies que se ubican en la categoría de muy baja densidad (Ochroma pyramidale, Pachira quinata). Es importante acotar que el menor diámetro de poros correspondió a la especie que mostró los menores valores de densidad (Ochroma pyramidale), mientras que el mayor valor de densidad correspondió a la especie con mayor frecuencia de poros (Catostemma commune).

Con relación a la subfamilia Grewioideae, las dos especies muestran diferencias estadísticamente significativas en los tres caracteres estudiados (Cuadro 3). En cuanto a los poros, de acuerdo a la clasificación de [38], Apeiba tibourbou se ubica en la categoría I y Heliocarpus americanus en la categoría II de frecuencia de poros mientras que en el diámetro de poros ambas especies se ubican en la categoría III. Los valores de densidad (Cuadro 3) ubican ambas especies en el grupo de maderas de densidad muy baja, de acuerdo a los criterios de [37], pero a pesar de estar incluidas en un mismo grupo, su comportamiento indica diferencias estadísticamente significativas.

La subfamilia Malvoideae estuvo representada por la especie Uladendron codesurii y fue la única que corresponde a Malvaceae sensu stricto de acuerdo al sistema de clasificación de Cronquist. El mayor valor de densidad seca al aire lo mostró ésta especie (Cuadro 3), ubicándose en la categoría $\vee$ de [37]. La frecuencia de poros promedio varió entre 6,77 poros/ $\mathrm{mm}^{2}$ y 11,15 poros $/ \mathrm{mm}^{2}$ (categoría II) y el diámetro de poros osciló entre 114,4 um y 144,5 $\mu \mathrm{m}$, ubicándose en la categoría III según [38]. Entre los dos individuos estudiados existieron diferencias significativas de acuerdo a la prueba de Tukey $(a=0,01)$, tanto en la frecuencia como en el diámetro de poros.

Con respecto a la subfamilia Sterculioideae (cuadro 3), la frecuencia y el diámetro de poros no mostraron diferencias entre Sterculia apetala y Sterculia pruriens, pero si mostraron diferencias en cuanto a la densidad seca al aire. Sterculia apetala se ubicó en el grupo de maderas de densidad muy baja y Sterculia pruriens en la categoría de baja densidad.

Al establecer comparaciones entre las diez especies estudiadas, se encontró una gran variabilidad (cuadro 4).

La frecuencia de poros (cuadro 4) osciló entre un mínimo aproximado de 3 poros $/ \mathrm{mm}^{2}$ (Ochroma pyramidale, Eriotheca globosa, Pachira quinata) hasta un máximo de 19 poros $/ \mathrm{mm}^{2}$ (Guazuma ulmifolia).

Por lo general, las menores frecuencias correspondieron a las subfamilias Bombacoideae y Sterculiodeae; mientras que los mayores valores se ubicaron en las subfamilias Grewioideae y Malvoideae. Según la clasificación de la [38] dos especies se ubican en la categoría I (Ochroma pyramidale, Pachira quinata); seis especies incluyen individuos en la categoría I y II (Catostemma commune, Eriotheca globosa, Apeiba tibourbou, Heliocarpus americanus, Sterculia apetala, Sterculia pruriens) y dos especies (Guazuma ulmifolia, Uladendron codesurii) se distribuyen en las categorías II y III. Los valores encontrados para frecuencia de poros coinciden con lo reportado para especies de Malvaceae por [6]; así como con lo indicado por [18] para Catostemma commune, Ochroma pyramidale, Pachira quinata, Guazuma ulmifolia, Sterculia apetala y Apeiba tibourbou; [40] en Pachira quinata; [12] para Apeiba tibourbou y Heliocarpus popayanensis; [40] para Catostemma commune, Eriotheca globosa, Ochroma pyramidale y Pachira quinata; [13] en Uladendron codesurii y lo encontrado por [42] en Catostemma commune. El diámetro de poros (Cuadro 4) mostró una alta variación y osciló entre un promedio mínimo de 129,45 $\mu \mathrm{m}$ (Uladendron codesurii) hasta un máximo de 286,5 $\mu \mathrm{m}$ (Pachira quinata), existiendo una diferencia superior al $100 \%$ entre ambos valores. 
Según la clasificación de la [38] todos los individuos de Pachira quinata y Sterculia apetala se ubican en la categoría de poros grandes (> $200 \mu \mathrm{m}$ ); mientras que Guazuma ulmifolia, Heliocarpus americanus y Uladendron codesurii tienen a todos sus individuos en la categoría de poros medianos (100 - $200 \mu \mathrm{m}$ ). El resto de las especies tienen individuos en las dos categorías antes mencionadas. Con relación a lo reportado por [6], [18], [40], [12], [41], [13], [42], y [43], existen similitudes con lo encontrado en las diez especies estudiadas con respecto a la categoría diamétrica del tamaño de los poros. A nivel de subfamilias, los poros de mayor tamaño se encontraron en Bombacoideae y Sterculioideae y los de menor tamaño en Grewioideae y Malvoideae. De acuerdo a la prueba de Tukey $(a=0,01)$ se puede considerar que Bombacoideae y Sterculioideae tienen un comportamiento similar y se pueden incluir en un mismo grupo. De acuerdo a los valores obtenidos en la frecuencia y diámetro de poros, se calculó la fracción de lumen de vasos para cada especie (Cuadro 5), encontrándose que el factor que mayor incidencia tiene sobre la fracción de lumen de vasos es la frecuencia de poros. Los mayores valores de fracción de lumen correspondieron a las especies con mayor frecuencia de poros (Guazuma ulmifolia, Heliocarpus americanus, Uladendron codesurii).

Cuadro 4. Comparación entre especies de la familia Malvaceae en frecuencia de poros, diámetro de poros y densidad seca al aire (promedio, desviación, coeficiente de variación, número de mediciones).

\begin{tabular}{|c|c|c|c|}
\hline Especie & Poros $/ \mathrm{mm}^{2}$ & Diámetro de poros $(\mu \mathrm{m})$ & Densidad seca al aire $\left(\mathrm{kg} / \mathrm{m}^{3}\right)$ \\
\hline Apeiba tibourbou & $\begin{array}{c}4,56^{\mathrm{abc}} \pm 1,43 \\
C V=31,36 \% ; n=20\end{array}$ & $\begin{array}{c}170,85^{c} \pm 28,81 \\
C V=16,86 \% ; n=100\end{array}$ & $\begin{array}{c}403^{b} \pm 51 \\
C V=12,62 \% ; n=40\end{array}$ \\
\hline $\begin{array}{l}\text { Catostemma } \\
\text { commune }\end{array}$ & $\begin{array}{c}4,85^{\mathrm{abc}} \pm 1,32 \\
\mathrm{CV}=27,19 \% ; n=20\end{array}$ & $\begin{array}{c}227,23^{e} \pm 38,23 \\
C V=16,82 \% ; n=100\end{array}$ & $\begin{array}{c}682^{d} \pm 90 \\
C V=13,15 \% ; n=40\end{array}$ \\
\hline Eriotheca globosa & $\begin{array}{c}3,42^{\mathrm{ab}} \pm 1,79 \\
\mathrm{CV}=52,43 \% ; n=20\end{array}$ & $\begin{array}{c}229,4^{\mathrm{e}} \pm 49,82 \\
C V=21,72 \% ; n=100\end{array}$ & $\begin{array}{c}607^{\circ} \pm 60 \\
C V=9,929 \% ; n=40\end{array}$ \\
\hline Guazuma ulmifolia & $\begin{array}{c}18,52^{d} \pm 11,07 \\
C V=59,78 \% ; n=25\end{array}$ & $\begin{array}{c}146,68^{\mathrm{ab}} \pm 24,67 \\
C V=16,82 ; n=125\end{array}$ & $\begin{aligned} 612^{\circ} \pm 61 \\
C V=9,92 \% ; n=50\end{aligned}$ \\
\hline $\begin{array}{l}\text { Heliocarpus } \\
\text { americanus }\end{array}$ & $\begin{array}{c}9,31^{d} \pm 6,85 \\
C V=73,59 \% ; n=20\end{array}$ & $\begin{array}{c}158,93^{\mathrm{bc}} \pm 29,98 \\
\mathrm{CV}=18,87 \% ; n=100\end{array}$ & $\begin{array}{c}230^{\mathrm{a}} \pm 43 \\
\mathrm{CV}=18,82 \% ; n=40\end{array}$ \\
\hline $\begin{array}{l}\text { Ochroma } \\
\text { pyramidale }\end{array}$ & $\begin{array}{c}3,04^{\mathrm{a}} \pm 1,7 \\
\mathrm{CV}=55,96 \% ; n=20\end{array}$ & $\begin{array}{l}193,55^{d} \pm 29,97(270) \\
C V=15,48 \% ; n=100\end{array}$ & $\begin{array}{c}185^{a} \pm 34 \\
C V=18,33 \% ; n=36\end{array}$ \\
\hline Pachira quinata & $\begin{array}{c}3,14^{a b} \pm 1,41 \\
C V=45,08 \% ; n=20\end{array}$ & $\begin{array}{c}286,45^{\dagger} \pm 62,18 \\
C V=21,71 \% ; n=100\end{array}$ & $\begin{aligned} & 436^{b} \pm 94 \\
C V= & 21,60 \% ; n=40\end{aligned}$ \\
\hline Sterculia apetala & $\begin{array}{c}4,04^{\mathrm{abc}} \pm 1,72 \\
\mathrm{CV}=42,53 \% ; n=20\end{array}$ & $\begin{array}{c}240,25^{e} \pm 29,85 \\
C V=12,43 \% ; n=100\end{array}$ & $\begin{aligned} & 457^{b} \pm 41 \\
C V= & 9,02 \% ; n=40\end{aligned}$ \\
\hline Sterculia pruriens & $\begin{array}{c}4,37^{a b c} \pm 2,32 \\
C V=53,19 \% ; n=20\end{array}$ & $\begin{array}{c}238,9^{e} \pm 45,76 \\
C V=19,16 \% ; n=100\end{array}$ & $\begin{aligned} & 597^{\circ} \pm 52 \\
C V= & 8,75 \% ; n=40\end{aligned}$ \\
\hline $\begin{array}{l}\text { Uladendron } \\
\text { codesurii }\end{array}$ & $\begin{array}{c}8,96^{b c} \pm 2,76 \\
C V=30,82 \% ; n=20\end{array}$ & $\begin{array}{c}129,45^{\mathrm{a}} \pm 23,27 \\
\mathrm{CV}=17,97 \% ; n=100\end{array}$ & $\begin{array}{c}1037^{e} \pm 100 \\
C V=9,62 \% ; n=11\end{array}$ \\
\hline
\end{tabular}

NOTA: Letras diferentes indican grupos con diferencias estadísticas significativas (Tukey, $a=0,01$ ). 
Cuadro 5. Frecuencia, diámetro y fracción de lumen de vasos para diez especies de Malvaceae.

\begin{tabular}{|c|c|c|c|}
\hline Especie & Poros $/ \mathrm{mm}^{2}$ & Diámetro de poros $(\mu \mathrm{m})$ & $\begin{array}{c}\text { Fracción de lumen de vasos }(\mu \mathrm{m} \times \\
\left.\mathrm{mm}^{2}\right)\end{array}$ \\
\hline Apeiba tibourbou & 4,56 & 170,85 & 779,08 \\
\hline Catostemma commune & 4,85 & 227,22 & 1102,02 \\
\hline Eriotheca globosa & 3,42 & 229,4 & 784,5 \\
\hline Guazuma ulmifolia & 18,52 & 146,67 & 2716,33 \\
\hline Heliocarpus americanus & 9,31 & 158,92 & 1479,54 \\
\hline Ochroma pyramidale & 3,04 & 193,55 & 588,39 \\
\hline Pachira quinata & 3,13 & 286,45 & 896,59 \\
\hline Sterculia apetala & 4,04 & 240,25 & 970,61 \\
\hline Sterculia pruriens & 4,37 & 238,9 & 1043,99 \\
\hline Uladendron codesurii & 8,96 & 129,45 & 1159,87 \\
\hline
\end{tabular}

La densidad seca al aire osciló entre $185 \mathrm{~kg} / \mathrm{m}^{3}$ (Ochroma pyramidale) y $1037 \mathrm{~kg} / \mathrm{m}^{3}$ (Uladendron codesurii) (cuadro 4), mostrando un rango de variación donde el mayor promedio registrado fue 5,6 veces superior al promedio mínimo.

De acuerdo con la clasificación de [37], el 50 \% de las especies se encuentran en la categoría I, $30 \%$ en la categoría II, $10 \%$ en la III y $10 \%$ en la categoría V. También se encontró que dentro de las categorías donde se ubicaron más de una especie (I, II) existieron diferencias estadísticamente significativas lo que indica que aún cuando se ubican en una misma categoría muestran diferencias de comportamiento en cuanto a densidad y esto puede incidir en diferencias en otras propiedades físicas y mecánicas. Con respecto a la ubicación de especies en las diferentes subfamilias, las de densidad muy baja a media pertenecen a las subfamilias Bombacoideae, Grewioideae, Byttnerioideae y Sterculioideae; mientras que la Malvoideae fue la única en la categoría de muy alta densidad. Al contrario de lo esperado, el mayor valor de densidad (Uladendron codesurii) coincidió con uno de los mayores valores de fracción de lumen de vasos; mientras que la menor densidad (Ochroma pyramidale) correspondió con la menor fracción de lumen de vasos. Al tener especies ubicadas en una misma categoría de densidad seca al aire pero que muestran diferencias significativas indica que la clasificación de [37] tal vez no es la más idónea para este grupo de Malvaceae y, tomando en consideración el análisis estadístico realizado para las diez especies, se puede establecer una clasificación en esta familia de acuerdo a los resultados obtenidos, sugiriéndose seis categorías de las cuales la I - II representan maderas blandas, la III - IV moderadamente duras y V - VI para maderas duras (cuadro 6).

Cuadro 6. Clasificación de densidad seca al aire (Dsa) para 10 especies de Malvaceae.

\begin{tabular}{|c|c|c|}
\hline Categoría & Dsa $\left(\mathrm{kg} / \mathrm{m}^{3}\right)$ & Especies \\
\hline I & $148-303$ & Ochroma pyramidale, Heliocarpus americanus \\
\hline II & $304-457$ & Pachira quinata, Apeiba tibourbou, Sterculia apetala \\
\hline III & $458-611$ & Eryotheca globosa, Sterculia pruriens \\
\hline IV & $612-765$ & Catostemma commune, Guazuma ulmifolia \\
\hline V & $766-919$ & --- \\
\hline VI & $920-1073$ & Uladendron codesurii \\
\hline
\end{tabular}


Al establecer relación entre los valores de densidad y de características de los vasos, la prueba de Pearson ( $a=0,01$ ) (cuadro 7) indica que existe una correlación significativa entre la frecuencia con el diámetro de poros y fracción de lumen de los vasos, observándose una relación directa entre frecuencia de poros y fracción de lumen e inversa entre frecuencia de poros y diámetro de poros, siendo mayor el nivel de significancia con la fracción de lumen de los vasos.

Cuadro 7. Correlación de Pearson entre densidad seca al aire (Dsa), frecuencia (Fp), diámetro (Dp) y fracción de lumen de vasos (Flv) para diez especies de Malvaceae.

\begin{tabular}{|c|c|c|c|c|c|c|}
\hline \multicolumn{2}{|c|}{} & Especie & $F p($ poros/mm²) & $\begin{array}{c}\text { Dp } \\
(\mu \mathrm{m})\end{array}$ & $\begin{array}{c}\text { Flv } \\
\left(\mu \mathrm{m} \times \mathrm{mm}^{2}\right)\end{array}$ & $\begin{array}{c}\text { Dsa } \\
\left(\mathrm{kg} / \mathrm{m}^{3}\right)\end{array}$ \\
\hline \multirow{3}{*}{$\mathrm{Fp}$} & Correlación de Pearson & 0,045 & 1 & $-0,526\left(^{* *}\right)$ & $0,962\left(^{* *}\right)$ & 0,183 \\
\cline { 2 - 7 } & Sig. (bilateral) & 0,784 & & 0,001 & 0,000 & 0,264 \\
\cline { 2 - 7 } & $\mathrm{n}$ & 39 & 39 & 39 & 39 & 39 \\
\hline \multirow{3}{*}{$\mathrm{Dp}$} & Correlación de Pearson & 0,049 & $-0,526\left(^{* *}\right)$ & 1 & $-0,334\left(^{*}\right)$ & $-0,029$ \\
\cline { 2 - 7 } & Sig. (bilateral) & 0,766 & 0,001 & & 0,038 & 0,863 \\
\cline { 2 - 7 } & $\mathrm{n}$ & 39 & 39 & 39 & 39 & 39 \\
\hline \multirow{3}{*}{ Flv } & Correlación de Pearson & 0,112 & $0,962\left(^{* *}\right)$ & $-0,334\left(^{*}\right)$ & 1 & 0,217 \\
\cline { 2 - 7 } & Sig. (bilateral) & 0,497 & 0,000 & 0,038 & & 0,185 \\
\cline { 2 - 7 } & $\mathrm{n}$ & 39 & 39 & 39 & 39 & 39 \\
\hline \multirow{3}{*}{ Dsa } & Correlación de Pearson & $-0,080$ & 0,183 & $-0,029$ & 0,217 & 1 \\
\cline { 2 - 7 } & Sig. (bilateral) & 0,629 & 0,264 & 0,863 & 0,185 & 39 \\
\cline { 2 - 7 } & $\mathrm{n}$ & 39 & 39 & 39 & 39 & 39 \\
\hline
\end{tabular}

** La correlación es significativa al nivel 0,01 (bilateral).

* La correlación es significante al nivel 0,05 (bilateral).

Con respecto a la densidad seca al aire, no se encontró correlación significativa con ninguno de los elementos cuantitativos de los vasos (frecuencia, diámetro, fracción de lumen); sin embargo presenta valores positivos con frecuencia de poros y fracción de lumen y negativos con diámetro de poros. Estos resultados contrastan con lo encontrado por [29] el cual reporta una relación negativa y significativa entre densidad y área o espacio ocupado por los vasos (incrementos de densidad ante disminuciones del área media de los vasos), además de una correlación positiva y significativa entre densidad de la madera y frecuencia de vasos. Asimismo, [22] y [32] encontraron correlaciones negativas para diámetro de poros y fracción de poros con la densidad de la madera, mientras que [44] reportan una correlación positiva entre diámetro de poros y densidad de la madera. Por otra parte, [24] señalan que la fracción de lumen y la densidad de la madera muestran una baja o nula correlación; tal como se encontró en el presente estudio con las diez especies de Malvaceae. Esto indica que los vasos, aunque ejercen influencia sobre la densidad de la madera, no explican por sí solos la variación de la densidad y existen otros tejidos xilemáticos que pueden tener mayor influencia y correlación con la densidad. Observando las características anatómicas de las especies estudiadas, se puede inferir que el grosor de paredes de las fibras puede tener una mayor influencia sobre la densidad; al contrastar los valores extremos se encuentra que la especie que mostró mayor valor de densidad seca al aire (Uladendron codesurii) se caracterizó por poseer fibras de paredes muy gruesas, mientras que los menores valores de densidad de Ochroma pyramidale coinciden con ser la especie que presentó fibras de paredes muy delgadas. Igualmente, Catostemma commune mostró el segundo valor de densidad más alto a pesar de tener poros grandes y de 
mayor dimensión que los de Ochroma pyramidale, pero también presentó fibras de paredes muy gruesas. [27], estudiando la madera de Avicennia marina, indican que aún cuando se presenten vasos grandes, la densidad de la madera es alta debido a la alta proporción del tejido leñoso que es ocupado por fibras de paredes gruesas.

\section{Conclusiones}

a) La densidad seca al aire en la Malvaceae mostró una variabilidad importante donde los valores encontrados van desde muy baja a media para las subfamilias Bombacoideae, Grewioideae, Byttnerioideae y Sterculioideae; mientras que la Malvoideae ha sido la única en la categoría de muy alta densidad según [37]. A partir del análisis estadístico (análisis de varianza y prueba de Tukey, $\alpha=0,01$ ) se elaboró una clasificación que presentó un comportamiento más acertado en cuanto a la agrupación de las especies y los resultados obtenidos para las diez especies estudiadas de esta familia.

b) La relación encontrada entre las características dimensionales de los elementos de conducción (frecuencia, diámetro, fracción de lumen), indicaron una correlación significativa. Para la densidad seca al aire no se encontró correlación significativa con ninguno de éstos elementos cuantitativos sin embargo presenta valores positivos con frecuencia de poros y fracción de lumen y negativos con diámetro de poros. Con esto se puede inferir que los otros elementos constitutivos del xilema (fibras y parenquima) son importantes para establecer relación entre la anatomía y la propiedad física de densidad.

\section{Referencias}

[1] L. Ninin. "La anatomía de la madera y la resolución de problemas tecnológicos". Rev Forest Venez 37: 107116. 1993.

[2] W. León. "Anatomía y densidad o peso específico de la madera". Rev Forest Venez 54(1): 67-76. 2010.

[3] APG. "An update of the Angiosperm Phylogeny Group classification for the orders and families of flowering plants: APG II". Bot J Linn Soc 141(4): 399-436. 2003.

[4] APG. "An update of the Angiosperm Philogeny Group classification for the orders and families of flowering plants: APG III”. Bot J Linn Soc 161(2): 105-121. 2009.

[5] APG. "An update of the Angiosperm Phylogeny Group classification for the orders and families of flowering plants: APG IV". Bot J Linn Soc 181(1): 1-20. 2016.

[6] L. Rojas y W. León. "Anatomía de la madera de 25 especies de Malvaceae en Venezuela”. Pittieria 43 : 8 - 33. 2019.

[7] J. Mora y J. Arroyo. "Propiedades físicas-mecánicas de 44 maderas de la Guayana Venezolana". Ministerio de Agricultura y Cría. Laboratorio Nacional de Productos Forestales. Mérida, Venezuela. 6 p. 1968.

[8] Laboratorio Nacional de Productos Forestales (LNPF). "Estudio de 104 maderas de los Altos Llanos Occidentales". Universidad de Los Andes. Facultad de Ciencias Forestales y Ambientales. Mérida, Venezuela. 1972.

[9] J. Lozada. "Propiedades físicas y mecánicas del Saquisaqui (Bombacopsis sepium) basadas en el estudio de una rola". Tesis de grado. Universidad de los Andes, Facultad de Ingeniería Forestal. Mérida, Venezuela. 1958.

[10] J. Merlo. E. Reyes. D. Garay y S. Valero. "Propiedades físico-mecánicas y usos posibles de la especie Eriotheca globosa (Aublet) A. Robyns (Ceiba roja). Proveniente del Lote Boscoso San Pedro, del estado Bolívar, Venezuela". Rev Forest Venez 48(1): 87-94. 2004.

[11] N. Angarita. "Estudio anatómico de algunas especies de la familia Bombacaceae". Trabajo de ascenso. Universidad de los Andes. Mérida, Venezuela. 45 p. 1981.

[12] L. Valverde y A. Rincón. "Anatomía de la madera de 3 especies de la familia Tiliaceae en Venezuela. (Apeiba tibourbou, Goethalsia meiantha y Heliocarpus popayanensis)". Agricultura Andina 14(1): 85-99. 2008. 
[13] L. Pinto. "Descripción anatómica del leño de Uladendron codesurii Marcano-Berti (Malvaceae)". Pittieria 7: 31-34. 1978.

[14] H. Corothie. "Estructura anatómica de 47 maderas de la Guayana venezolana y clave para su identificación". Universidad de Los Andes. Laboratorio Nacional de Productos Forestales. Mérida, Venezuela. 125 p. 1967.

[15] A. Pérez. "Estructura anatómica de 37 maderas de la Guayana venezolana y clave para su identificación". Acta Bot Venez 8(1): 9-109. 1973.

[16] A. Pérez. "Anatomía e identificación de 40 maderas del bosque La Mucuy, estado Mérida". Pittieria 20: 5-78. 1993.

[17] W. León. "Anatomía de la madera de 69 especies de la Reserva Forestal Caparo (Barinas, Venezuela)". Rev Forest Venez 56(2): 167-195. 2012.

[18] W. León. "Anatomía de maderas de 108 especies de Venezuela". Pittiera. Serie Publicaciones Especiales PE 1. Mérida, Venezuela. 263 p. 2014.

[19] W. León. "Anatomía xilemática de tronco y ramas de Theobroma cacao L. (Malvaceae: Byttneroideae)". Ernstia 25(1): 1-17. 2015

[20] S. Aguilar, L. Abundiz y J. Barajas. "Comparación de la gravedad específica y características anatómicas de la madera de dos comunidades vegetales en México". Anales Inst Bio. Univ Nac Autón México, Bot 72(2): 171-185. 2001.

[21] G. Bárcenas, F. Ortega, G. Ángeles y P. Ronzón. "Relación estructura-propiedades de la madera de angiospermas mexicanas". Universidad y Ciencia 21(42): 45-55. 2005.

[22] K. Preston, W. Cornwell y J. DeNoyer. "Wood density and vessel traits as distinct correlates of ecological strategy in 51 California coast range angiosperms". New Phytol 170(4): 807-818. 2006.

[23] R. Moya, A. Berrocal, J. Serrano y M. Tomazello. "Variación radial de la anatomía, densidad y durabilidad de la madera de teca (Tectona grandis) procedente de dos calidades de 17 sitios y dos regiones climáticas de Costa Rica". Invest Agrar: Sist Recur For 18(2): 119-131. 2009.

[24] A. Zanne, M. Westoby, D. Falster, D. Ackerly, S. Loarie, S. Arnold y D. Coomes. "Angiosperm wood structure: global patterns in vessel anatomy and their relation to wood density and potential conductivity". Am J Bot 97(2): 207-215. 2010.

[25] V. Interian-Ku, M. Borja, V. de la Rosa, J. Garcia, A. Romero y H. Vaquera. "Características anatómicas y propiedades físicas de la madera de Caesalpinia gaumeri Greenm en Dzan, Yucatán". Madera y Bosques 17(1): 23-36. 2011.

[26] P. Pande y R. Dhiman. "Variations in wood anatomical properties and specific gravity of half sib progenies of Populus deltoids". J For Res 23(3): 491-496. 2012.

[27] N. Santini, N. Schmitz y C. Lovelock. "Variation in wood density and anatomy in a widespread mangrove species. Trees 26(5): 1555-1563. . 2012.

[28] S. Monteoliva y T. Cerrillo. "Densidad y anatomía de la madera en familias mejoradas de sauces en argentina". Rev Árvore 37(6): 1183-1191. 2013.

[29] M. Strubbe. "Variation of wood density and vessel traits along an altitude gradient in a tropical montane cloud forest in Ecuador". Master thesis. Universiteit Gent, Faculty of Bioscience Engineering, Laboratory of plant ecology \& laboratory of wood technology. Belgium. 2013. (https://lib.ugent.be/en/catalog/rug01:002063423).

[30] C. Puchaicela. "Estudio de la estructura anatómica y propiedades físico-mecánicas de cinco especies maderables en bosques secundarios del cantón Zamora". Tesis de grado. Universidad Nacional de Loja. Loja, Ecuador. 161 p. 2013. (https://dspace.unl.edu.ec/jspui/handle/123456789/5218).

[31] S. Monteoliva, A. Barotto y M. Fernández. "Anatomía y densidad de la madera en Eucalyptus: variación interespecífica e implicancia en la resistencia al estrés abiótico". Rev Fac Agron Univ Nac La Plata 114(2): 209-217. 2015.

[32] S. Monteoliva, A. Barotto, P. Alarcón, N. Tesón y M. Fernández. "Densidad de la madera como variable integradora de la anatomía del leño: análisis de ramas y fuste en cuatro especies de Eucalyptus". Rev Fac Agron Univ Nac La Plata 116(1): 1-11. 2017.

[33] N. Espinoza y W. León. "Influencia de las características anatómicas sobre las propiedades mecánicas de la madera”. Rev Forest Venez 37: 21-38. 1993.

[34] A. Taquire. "Relación entre la estructura anatómica y las propiedades físico mecánicas de tres especies forestales provenientes de los altos Ilanos occidentales de Venezuela". Tesis de Maestría. Universidad de los Andes, Facultad de Ciencias Forestales y Ambientales. Mérida, Venezuela. 67 p. 1995. 
[35] S. Valero. "Relación entre anatomía y propiedades físico-mecánicas de la especie Tectona grandis, proveniente de los Llanos Occidentales de Venezuela". Tesis de Maestría. Universidad de los Andes, Facultad de Ciencias Forestales y Ambientales. Mérida, Venezuela. 101 p. 2001.

[36] Deustche Institut fur Normung (DIN). "Testing of wood; determination of density". German National Standard. DIN 52182. 3 p. 1976.

[37] P. Ninin. "Lineamientos Básicos Para la Utilización de Maderas". Tesis de grado. Universidad de Los Andes. Mérida, Venezuela. 256 p. 1987.

[38] IAWA Committee. "IAWA List of microscopic features for hardwood identification". IAWA Bull n.s. 10(2): 219332. 1989.

[39] A. Scholz, M. Klepsch, Z. Karimi y S. Jansen. "How to quantify conduits in wood?". Front Plant Sci 4: 1-11. 2013. DOI: 10.3389/fpls2013.0056.

[40] N. Barrios, W. León y C. Pacheco. "Determinación de características anatómicas cuantitativas de la madera mediante tecnologías de información geográfica". Pittieria 40: 54-93. 2016.

[41] P. Détienne, A. Loureiro y P. Jacquet. "Estudo anatómico do lenho da familia Bombacaceae da América". Acta Amaz 13(5-6): 831-867. 1983.

[42] E. Paula. "Estudos sobre Bombacaceae: Investigacao anatomica das madeiras de Catostemma commune Sandwith, Catostemma sclerophyllum Ducke e Scleronema micranthum (Ducke) Ducke, com vistas a polpa, papel e taxinomia". Acta Amaz 6(2): 155-161. 1976.

[43] D. Kribs. "Commercial foreign woods on the american market". Dover Publications Inc. New York. 241 p. 1968.

[44] L. Poorten, I. McDonald, A. Alarcon, E. Fichtler, J. Llcona, M. Peña, F. Sterck, Z. Villegas y U. Sass-Klaassen. "The importance of wood traits and hydraulic conductance for the performance and life history strategies of 42 rainforest tree species". New Phytol 185(2): 481-492. 2010. 